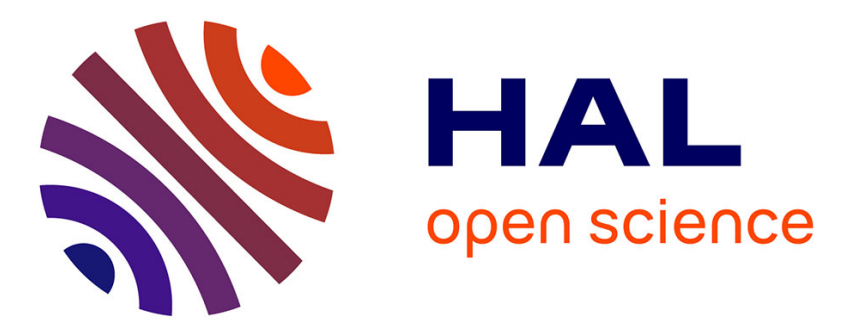

\title{
Accounting for Patterns of Collective Behavior in Crowd Locomotor Dynamics for Realistic Simulations
}

\author{
Stéphane Bonneaud, Kevin Rio, Pierre Chevaillier, William H Warren
}

\section{To cite this version:}

Stéphane Bonneaud, Kevin Rio, Pierre Chevaillier, William H Warren. Accounting for Patterns of Collective Behavior in Crowd Locomotor Dynamics for Realistic Simulations. The 24th International Conference on Computer Animation and Social Agents (CASA 2011), May 2011, Chengdu, China. pp.1-11, 10.1007/978-3-642-29050-3_1. hal-01169429

\section{HAL Id: hal-01169429 \\ https://hal.science/hal-01169429}

Submitted on 29 Jun 2015

HAL is a multi-disciplinary open access archive for the deposit and dissemination of scientific research documents, whether they are published or not. The documents may come from teaching and research institutions in France or abroad, or from public or private research centers.
L'archive ouverte pluridisciplinaire HAL, est destinée au dépôt et à la diffusion de documents scientifiques de niveau recherche, publiés ou non, émanant des établissements d'enseignement et de recherche français ou étrangers, des laboratoires publics ou privés.

\section{()ㅜ) $\Theta$}

Distributed under a Creative Commons Attribution - NoDerivatives| 4.0 International 


\title{
Accounting for Patterns of Collective Behavior in Crowd Locomotor Dynamics for Realistic Simulations
}

\author{
Stéphane Bonneaud ${ }^{1}$, Kevin Rio ${ }^{1}$, Pierre Chevaillier $^{2}$, and William H. Warren ${ }^{1}$ \\ 1 Dept. of Cognitive, Linguistic and Psychological Sciences, Brown University, USA \\ 2 ENIB-UEB; LISYC: Computer Science Laboratory for Complex Systems, France
}

\begin{abstract}
Do people in a crowd behave like a set of isolated individuals or like a cohesive group? Studies of crowd modeling usually consider pedestrian behavior either from the point of view of an isolated individual or from that of large swarms. We introduce here a study of small crowds walking towards a common goal and propose to make the link between individual behavior and crowd dynamics. Data show that participants, even though not instructed to behave collectively, do form a cohesive group and do not merely treat one another as obstacles. We present qualitative and quantitative measurements of this collective behavior, and propose a first set of patterns characterizing such behavior. This work is part of a wider effort to test crowd models against observed data.
\end{abstract}

Keywords: locomotion dynamics, collective behavior, crowd simulation.

\section{Introduction}

Crowd models for virtual worlds need to be persuasive, which can be achieved by developing (1) more plausible behavioral animation models, (2) richer models of the environment or (3) more efficient crowd rendering techniques [16, 8, 22]. Our interest here lies in building more reliable behavioral animation models of locomotor behavior for pedestrians that aggregate into crowds.

We advocate that, before developing new crowd simulation models, there is a need to better understand how collective behavior can emerge from the individual locomotion behaviors of pedestrians aggregating in a crowd. This necessary first step requires the identification of reproducible behavioral patterns from observations and systematic measures of individual behavior. By rigorously identifying the patterns that simulations should account for, psychologists can help computer animation go beyond the limitations of existing crowd simulations.

Most crowd simulation studies focus either on isolated pedestrians and their behaviors in virtual cities [9] or on large swarms and large-scale self-organisation phenomena $[10,8]$. However, how collective behavior emerges from interactions among individual pedestrians is still not fully understood [15]. Very few studies have focused on the modelling of local interactions of pedestrians building up 
small groups and most agent-based models of crowd dynamics $[11,18]$ have not been tested against field observations of human behavior [12]. [14] has studied the behavior of a pedestrian walking in a corridor and interacting with a stationary pedestrian and with another pedestrian walking in the opposite direction, but these are not common situations in crowd simulation and do not explain selforganisation phenomena. More recently, [15] emphasized the need to empirically study pedestrian groups, showing that social groups are very common in crowds and that crowd density impacts these groups' spatial patterns.

This paper presents the analysis of the results obtained from a cognitive psychology experiment on small crowds aimed at understanding whether and how individuals form a cohesive group when walking towards a common goal. These results allow us to identify patterns that could be explained by general laws and that could be later implemented into crowd simulation models for computer animation. The main objective here is to identify invariants in pedestrian behavior, namely locomotion behaviors, and not to introduce individual variability in crowd simulation, which is another issue [21]. We investigate the impact of density on a small crowd of participants, in order to make the link between individual locomotion and crowd dynamics. If individuals exhibit collective behavior in these conditions, then the phenomenon might help explain larger crowd dynamics. While identifying collective behavior at this level and in a controlled environment is a result in itself, it also allows us to develop measures of such behavior and to extract patterns that characterize human crowds.

In this article, we first contextualize the need for empirical data in the computer animation field. Second, we describe the behavioral experiment that was conducted and the resulting observations. Finally, we identify patterns characterizing collective behavior and the control variables that might yield coherent crowds. We argue that this knowledge is crucial for the computer animation field and could be used to develop and validate computational models of crowd dynamics.

\section{Crowd Simulation}

The best way to produce realistic simulations of crowds is to develop behavioral models that account for what real pedestrians do, and validate these models against observations of real pedestrians. Many studies have tried to understand how nearly all species can locomote and navigate in space with no apparent effort $[6,20,3]$. More specifically, humans walk in groups, avoid moving or stationary obstacles, and steer to common goals, while being involved at the same time in other cognitive activities, like social interactions with other members of the group. According to this theoretical framework [6,24], it should be possible to identify typical patterns resulting from these fundamental behavioral rules.

Surely, from a computer animation perspective, it is necessary to obtain agent-based simulations that (1) account for complex scenarios, e.g. interacting with a street artist while in a crowd, and (2) avoid forbidden behaviors, e.g. walking through walls or colliding with other pedestrians. Agent architectures and 
complex technological solutions have been proposed for generating such behaviors. Several models of locomotion are thus based on cognitive maps and internal models of the environment that enable the agent to calculate an optimal route and avoid collisions in a given local environment [23,17]. [19] have proposed a solution inspired from social psychology, but advocated obtaining unnatural motions at the micro level. Generally speaking, one of the main issues with such approaches is that these models have many parameters and thus are difficult to calibrate. Others try to use videos and learning algorithms to either automatically extract behavioral rules of locomotion in crowds [13] or to calibrate their models [14]. But this research, though very promising, has yet to come up with definitive solutions. Unfortunately, research in psychology or sociology cannot yet solve these problems because we don't know the control laws that actually govern human locomotion.

More reactive approaches have been proposed, based on the assumption that the agent's trajectories in space emerge from the interactions between the agent and its local environment without any internal representation of it [1]. Following such an approach, $[24,4,5]$ have produced a model of individual locomotion that precisely accounts for individual trajectories in space in various scenarios. This model has a number of advantages, namely: (1) it is grounded in a clear theoretical framework; (2) it explains what control variables and strategies are used by humans when they locomote in space and interact with moving or stationary targets and obstacles; (3) it is low parameterized, as the same few processes and parameters are used for each of the scenarios the model accounts for. Although the model of $[4,5]$ only accounts for simple scenarios thus far, making its use in computer animation not straightforward, we argue that this approach is currently the most relevant one to obtain validated simulations that can account for general laws of collective behaviors in crowds and thus allows us to identify typical behavioral patterns.

\section{Experiment}

\subsection{Objective}

The experiment aims at understanding the formation and structure of small groups of pedestrians walking towards a common goal; more specifically, we want to determine whether participants behave as a set of individuals or a coherent group, and how to characterize such collective behavior. We further wish to understand the control variables that govern local interactions among participants, and may underlie group formation. The independent variables were (1) the initial density of the group, characterized by the initial distance between participants, and (2) the initial bearing angle between the goal and the participants.

\subsection{Apparatus}

The experiment was built upon the approach and method of $[4,5]$. The experiment setup (Fig. 1) was based on experiment 1A of [4] that was designed to 
study how a single pedestrian steers towards a stationary target. The only difference in the setup is that we now consider four individuals walking together, instead of one.

Participants walked freely in a $12 m \times 12 m$ room. Their task was to walk to one of three goals (Fig. 1) specified out loud to all participants after the beginning of each trial. The goals were represented by poles of $\sim 2 \mathrm{~m}$ high and $\sim 30 \mathrm{~cm}$ wide. Participants were initially positioned in a "square" shape, whose side length characterized the initial density. As shown in the figure, there were four conditions for the initial density (x-small, .5m; small, 1m; wide, $1.5 \mathrm{~m}$; and $\mathrm{x}$-wide, $2.5 \mathrm{~m}$ ) and three possible goals.

For each trial, participants were first arranged in a randomly chosen configuration (density). Experimenters told each participant to stand on top of a number on the floor, announcing them out loud. Note that experimenters never spoke directly about density or the global "square" shape, but only spoke to participants as individuals, giving them information specific to each of them individually. Then, participants were told to start walking at a self-selected "comfortable pace" in a straight line. Once the last participant had crossed an invisible line $1 \mathrm{~m}$ from the front of the group (the dashed line in Fig. 1), an experimenter directed the participants to one randomly selected goal. Participants were instructed to walk to the goal and remain in contact with it (e.g. by keeping a hand on it). When all four participants were in contact with it, an experimenter asked them to walk back to other randomly chosen starting locations.

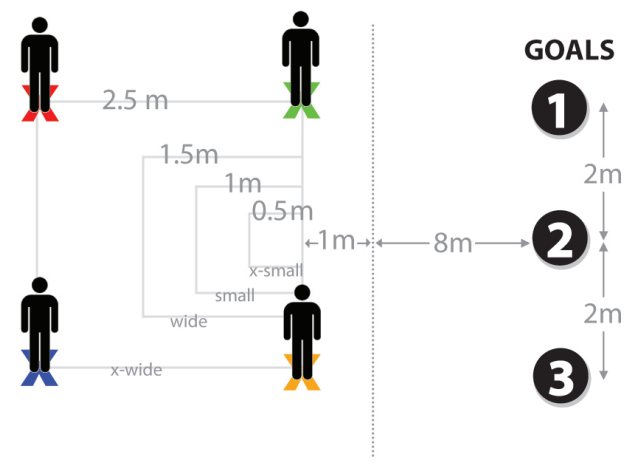

Fig. 1. Experimental setup: initial spatial setup of participants and goals.

There were 12 conditions: 4 densities $\times 3$ goals. Five groups of four participants were studied, with each group receiving 8 trials per condition (total: 96 trials). For each of those 8 trials, the initial locations of the participants were randomized, so that each density condition was tested with participants at different positions in the "square" shape. There were 20 participants (12 female), with a mean age of 23.35 years old $(S D=5.2)$. Participants did not know each other and groups were always mixed. Participants' head positions $(4 \mathrm{~mm}$ 
root-mean-square error) were measured by a hybrid inertial-ultrasonic tracking system (IS-900, Intersense, Burlington, MA) with six degrees of freedom, at a sampling rate of $60 \mathrm{~Hz}$.

\subsection{Definitions}

Position in group - The initial "square" shape had four relative positions defined with respect to the walking direction of the group: front-left, front-right, back-left and back-right. The goal can be characterized in the same way. The same reference system is used to characterize the direction of the group and the direction of the goal. With such reference system, participants walking towards the goal see the back of it.

Heading - A participant's heading or direction of travel was computed from the walking trajectory (rather than from head orientation data). The heading at time $t$ is calculated using the participant's positions at time $t$ and $t+1$.

Alone vs group speed - Participants were asked to walk on their own, one at a time, to goal 2 before starting the experiment. In this way, we obtained a baseline measure of each participant's self-selected "comfortable pace". These speeds are designated here as the alone speeds as opposed to speeds in group.

Interpersonal distance - The density of the group is characterized by the distances between participants. The interpersonal distance of the group (or average individual interpersonal distance) is here the mean of all six distances between each participant. We also computed the average anterior-posterior $(\sim$ back to front) distance and the average lateral ( $\sim$ shoulder to shoulder) distance.

Collective behavior - This term is used here to contrast with more individualistic behavior: where isolated pedestrians may simply avoid other pedestrians as if they were obstacles, pedestrians exhibiting collective behavior show evidence of synchronizing their behaviors and the system at the group level exhibits stable patterns.

\subsection{Results}

Individual Behaviors. First, participants were consistent throughout the trials and they tended to maintain the initial group structure throughout trials. That is, participants' positions in the group were stable as shown in Fig. 2 and, overall, the group's geometry was stable as well: e.g. an individual starting at the front-right in the group had the tendency to have its ending position on the front-right side of the goal.

Second, while alone speeds were significantly different across participants, individuals adjusted their speed when walking in a group: specifically, they tended to walk slower when in a group $(M=1.02 \mathrm{~m} / \mathrm{s})$ than when alone $(M=1.09$ $\mathrm{m} / \mathrm{s}), t(15)=2.36, p<.05$. These adjustments are more pronounced for faster individuals, as shown in Fig. 3. There is a negative correlation $(r(14)=-.842$, $p<.001$ ) between an individual's preferred speed and their adjustment when in a group (i.e. the difference between their group and alone speeds). 


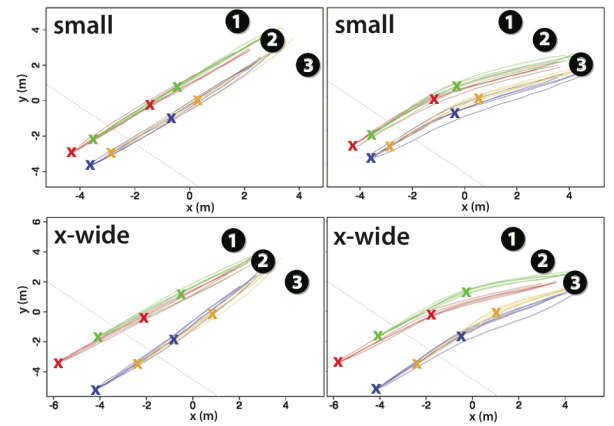

Fig. 2. Each line is an average of 8 individual trajectories of one group for a given "position" in the group (e.g. top left); for each "position" (each color), there are 5 lines for the 5 groups. Initial positions and positions at half time of the trial are designated by the crosses.

Third, individual heading trajectories were consistent across participants as shown in Fig. 4. The figure shows that the variability of the average heading for each condition is low, which is strong evidence that participants had a tendency to steer towards the goal consistently with the group. Note that the heading's dynamics seem to be alike whatever the initial density of the group.

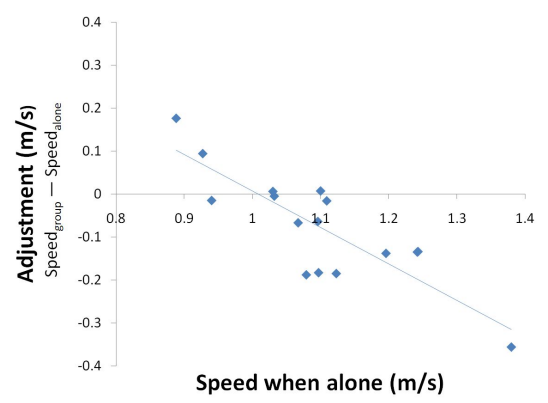

Fig. 3. Difference between the group speeds and alone speeds against the alone speeds for each participant.

Group Dynamics. In Fig. 5, the graph on the left shows the evolution in time of the mean interpersonal distance per condition. The x-small, small and wide configurations exhibit a convergence towards distances in between 1.3 and 1.6 meters. This could represent a preferred or stable density of the group. In the wide configuration, the mean interpersonal distance seems stable during most of the trial before dropping when participants reach the goal. In the x-small configuration, the distance first increases very quickly as participants, in order not 

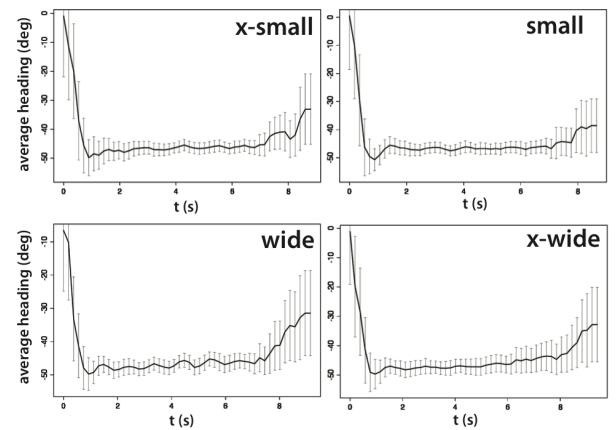

Fig. 4. Average heading dynamic in time for goal 2 and per density. The vertical lines show the standard error.

to collide with each other, spread out and double the initial $.5 m$ that separates them. After this quick expansion, the distance continues to increase more slowly, and the distance's dynamic seems to be closer to that observed in small configuration. In the small configuration, the distance appears to increase slightly to reach a more stable density close to that observed in the wide configuration. In the $\mathrm{x}$-wide configuration, the distance never stabilizes, but continues to contract to the goal. It is not clear whether the distance would stabilize itself around $1.5 \mathrm{~m}$ if the goal was further away.

Finally, Fig. 5 shows that the dynamics of the anterior-posterior and lateral distances are quite similar, implying that the same processes could control them. The anterior-posterior distance tends towards a larger preferred value than the lateral distance. Presumably, the anterior-posterior distance is controlled by the participant in back, whereas the lateral distance is controlled by both members of a pair. Nevertheless, the anterior-posterior variability is no greater than the lateral variability.
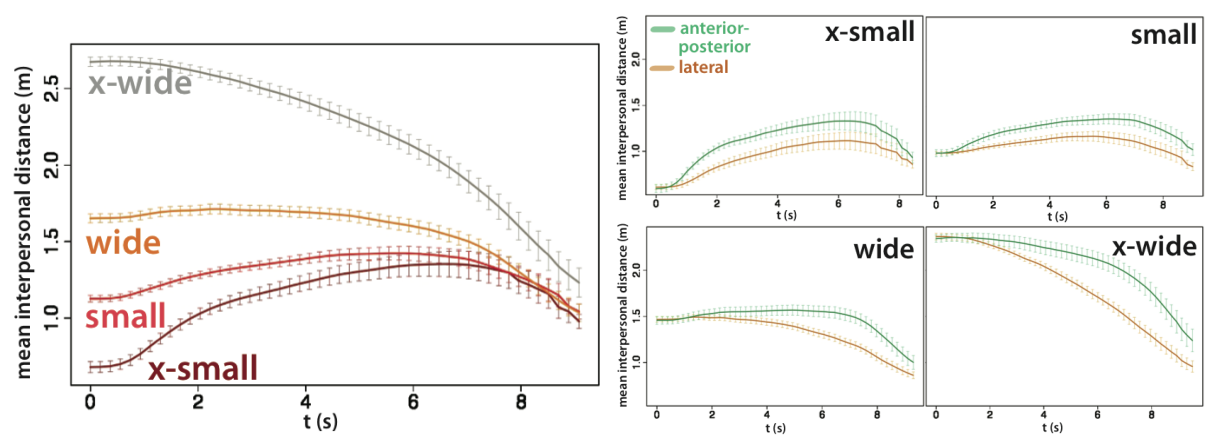

Fig. 5. Interpersonal distance per condition through time. The vertical lines show the standard error. 


\section{Patterns of Collective Behavior}

\subsection{Analysis}

A first remark for crowd simulation is that the group's consistency in structure and dynamic, of the heading and speeds, is a strong sign that the modeling of the phenomenon is accessible and that the patterns are reliably identifiable.

Second, the most important result of this study is that participants do not seem to behave like isolated pedestrians, but exhibit collective behavior. This is a clear result that has not yet been shown in a small crowd, with participants who do not know each other and with no explicit instruction to exhibit such behavior.

Strong evidence of collective behavior appears in the results on speed and on interpersonal distances. Consistency of the group's dynamic is suggestive of collective behavior, although it might be explained by the physical constraints of the task itself. Seen through the stability of the geometry of the group, the consistency also reveals how the group self-organizes upon arrival at the goal. The first paragraph of section 3.4 shows how individuals tend to keep their positions in the group and organize themselves around the goal as they were organized in the group. Findings of [4] show that a single individual goes directly on the goal. Here, the task does partly explain their distribution around the goal, and one could argue that it is easier for participants to reach the goal in such a manner. However, participants' trajectories are very direct towards these pre-selected positions (or sub-goals) around the goal.

\subsection{Patterns}

Group structure: the group exhibits consistency and little variability (visible in the dynamics of the heading and structure or geometry of the group);

Speed synchronization: individuals adopt a common speed; this speed is closer to the slower individuals so that faster individuals change their speed more;

Preferred density: individuals adjust their interpersonal distances in order to approach a stable density between 1.3 and $1.6 \mathrm{~m}$;

Anisotropy of interpersonal distances: individuals tend to keep a greater distance from others that are in front or behind, than from those that are next to them.

\section{Discussion}

This study shows that pedestrians, when surrounded by other pedestrians going to the same goal, tend to modify their behaviors according to others and exhibit collective behavior. They tend to adjust their speed to coordinate with others, adopting a common speed that is closer to the speeds of the slowest individuals. The structure of the group tends to maintain a stable "rectangular" shape, with 
a smaller shoulder-to-shoulder distance, leaving a bigger front-to-back distance. More strikingly, the density of the group tends to converge towards a preferred stable value, which is very clear for the $\mathrm{x}$-small, small and wide configurations. Low variabilities of the standard errors for the interpersonal distances show that those results are consistent across trials and groups, and confirm the structure of the group. Even though the task itself constrains the behavior of the individuals, such clear self-organization with only four people was not implied by the instructions or the simple nature of the task. Finally, the similar dynamics and comparably low variability of the front-and-back and shoulder-to-shoulder distances may imply that the same control variables are being used to organize both distances.

As with many psychological experiments, the experimental setup constrained the participants to a task in order to make systematic observations in a controlled environment. To a certain extent, these constraints might force individual trajectories into dynamics that could, after analysis, be mistakenly interpreted as collective behavior. The initial positioning of participants in a "square" shape and the initial distance to the goal (less than $10 \mathrm{~m}$ ) are two strong constraints. Concerning the initial positioning, observational studies [2] show that, when in groups, pedestrians tend to walk two by two, so the configuration is reasonable in this context. Concerning the distance to the goal, one limitation of the setup is that a longer distance would emphasize the stability of the observed dynamics. Yet, steering to a goal is a very general and common activity in crowd locomotion. Observing collective behavior and behavioral synchronization in such a short time and distance show the rapid and pronounced impact of other pedestrians on individual behavior. Also, in a few trials, participants switched positions (e.g. some participants passed those in front) showing that the task was not so constrained. And longer paths alone would not guarantee that participants would be less socially constrained. As such, the experimental setup does enable us to isolate patterns of collective locomotion and to answer the question of this article.

From the computer animation point of view, this bridging between data and models, cognitive science and computer animation, can lead to better models, based on or inspired by the patterns of collective behavior shown here. We argue that such work is a necessary step towards a full understanding of collective behavior in large crowds. Crowd simulations dramatically need validation and testing against empirical observations. Patterns are the best way to do this [7]. But, such patterns also give evidence of the control variables humans might use, therefore helping make modeling assumptions. Much work remains to be done to clearly and fully identify the behavioral strategies used by humans that give rise to such collective behavior.

\section{Conclusion}

Our focus here was to address whether individuals act as isolated pedestrians or as a cohesive group when locomoting with others towards a common goal. 
We presented an experiment on small crowds of four pedestrians, not instructed to act collectively, in order to bridge between individual locomotion and crowd dynamics. The experiment was built based on $[4,5]$ who studied individual locomotion. This approach enabled us to show that individuals self-organize into groups, maintaining a consistent group structure, while adopting a common speed and a preferred interpersonal density. We have identified patterns that can be used to build and validate models of crowd dynamics and help identify the control variables that govern the emergence of such collective behavior.

The next step is threefold. First, it is necessary to analyse to what extent existing computational models of pedestrian locomotion could account for the patterns we have identified, determine their sensitivity and robustness for a given set of parameter values, and consider how they could be extended to better fit the experimental data. Second, additional experiments need to be performed in order to improve our list of patterns. Third, laboratory data has to be compared to ecological data. Laboratory conditions offer controlled and regular trajectories, a systemic study of behavior, and clear patterns. And, most likely, individuals use the same environmental information and behavioral strategies to interact with others whatever the conditions. If anything is different, we believe that it might be the level of activation of each behavioral strategy, i.e. their parametrization, making the patterns still valid, but necessitating comparison with ecological data.

Acknowledgments. This work was supported by the NIH grant R01 EY010923.

\section{References}

1. Brooks, R.A.: Intelligence without representation. Artificial Intelligence 47, 139159 (1991)

2. Costa, M.: Interpersonal distances in group walking. J Nonverbal Behav 34, 15-26 (2010)

3. Duchon, A., Warren, W.: A visual equalization strategy for locomotor control: Of honeybees, robots, and humans. Psychological Science 21, 183-202 (2002)

4. Fajen, B.R., Warren, W.H.: Behavioral dynamics of steering, obstacle avoidance, and route selection. Journal of Experimental Psychology: Human Perception and Performance 29, 343-362 (2003)

5. Fajen, B.R., Warren, W.H.: Behavioral dynamics of intercepting a moving target. Exp. Brain Res. 180, 303-319 (2007)

6. Gibson, J.J.: Visually controlled locomotion and visual orientation in animals. Ecological Psychology 10, 161-176 (1998), (Reprinted from British Journal of Psychology, 49, 182-194, 1958)

7. Grimm, V., Revilla, E., Berger, U., Jeltsch, F., Mooij, W.M., Railsback, S.F., Thulke, H.H., Weiner, J., Wiegand, T., DeAngelis, D.L.: Pattern-oriented modeling of agent-based complex systems: lessons from ecology. Science 310, 987-991 (2005)

8. Heigeas, L., Luciani, A., Thollot, J., Castagné, N.: A physically-based particle model of emergent crowd behaviors. In: Graphicon (2003), http://artis.imag. $\mathrm{fr} /$ Publications/2003/HLTC03 
9. Helbing, D.: A mathematical model for the behavior of pedestrians. Behavioral Science 36, 298-310 (1991)

10. Helbing, D.: Modelling the evolution of human trail systems. Nature 388, 47-50 (1997)

11. Helbing, D., Molnár, P.: Social force model for pedestrian dynamics. Physical Review E 51, 4282-4286 (1995)

12. Lakoba, T., Kaup, D., Finkelstein, N.M.: Modifications of the Helbing-MolnárFarkas-Vicsek social force model for pedestrian evolution. SIMULATION 81, 339 $352(2005)$

13. Lee, K.H., Choi, M.G., Hong, Q., Lee, J.: Group behavior from video: a datadriven approach to crowd simulation. In: ACM SIGGRAPH/Eurographics symposium on Computer Animation (2007), http://portal.acm.org/citation.cfm? id $=1272706$

14. Moussaid, M., Helbing, D., Garnier, S., Johansson, A., Combe, M., Theraulaz, G.: Experimental study of the behavioural mechanisms underlying self-organization in human crowds. In: Proceedings of the Royal Society B (2009)

15. Moussaid, M., Perozo, N., Garnier, S., Helbing, D., Theraulaz, G.: The walking behaviour of pedestrian social groups and its impact on crowd dynamics. PloS ONE 5(4), e10047 (2010)

16. Musse, S.R., Thalmann, D.: Hierarchical model for real time simulation of virtual human crowds. IEEE Transactions on Visualization and Computer Graphics 7, 152-164 (2001)

17. Paris, S., Pettré, J., Donikian, S.: Pedestrian reactive navigation for crowd simulation: a predictive approach. EUROGRAPHICS, Computer Graphics Forum 26, 665-674 (2007)

18. Reynolds, C.W.: Steering behaviors for autonomous characters. Game Developers Conference [online] (1999)

19. Sakuma, T., Mukai, T., Kuriyama, S.: Psychological model for animating crowded pedestrians. Computer Animation \& Virtual Worlds 16(3-4), 343-351 (2005), http://doi.wiley.com/10.1002/cav. 105

20. Schoner, G., Dose, M.: A dynamical systems approach to task-level system integration used to plan and control autonomous vehicle motion. Robotics and $\mathrm{Au}-$ tonomous Systems 10, 253-267 (1992)

21. Thalmann, D., Grillon, H., Maim, J., Yersin, B.: Challenges in crowd simulation. In: Proceedings of the 2009 International Conference on CyberWorlds. pp. 1-12. Washington, DC, USA (2009), http://dx.doi.org/10.1109/CW.2009.23

22. Thalmann, D., Musse, S.R.: Crowd simulation. Springer (2007), http://books . google.com/books?id=-aY5V-ykawcC

23. Thomas, R., Donikian, S.: A spatial cognitive map and a human-like memory model dedicated to pedestrian navigation in virtual urban environments. Lecture Notes in Computer Science 4387/2007, 421-438 (2007)

24. Warren, W.H., Fajen, B.R.: Understanding Complex Systems, chap. Behavioral Dynamics of Visually Guided Locomotion, pp. 45-75. Springer Berlin / Heidelberg (2007) 